\title{
On the precision of a length measurement
}

\author{
X. Calmet ${ }^{\mathrm{a}, \mathrm{b}}$ \\ Université Libre de Bruxelles, Service de Physique Théorique, CP 225, Boulevard du Triomphe, 1050 Bruxelles, Belgium
}

Received: 19 July 2007 / Revised version: 2 January 2008 /

Published online: 7 February 2008 - (C) Springer-Verlag / Società Italiana di Fisica 2008

\begin{abstract}
We show that quantum mechanics and general relativity imply the existence of a minimal length. To be more precise, we show that no operational device subject to quantum mechanics, general relativity and causality could exclude the discreteness of spacetime on lengths shorter than the Planck length. We then consider the fundamental limit coming from quantum mechanics, general relativity and causality on the precision of length measurement.
\end{abstract}

PACS. 04.20.-q; 03.65.-w

\section{Introduction}

Twentieth century physics has been a quest for unification. The unification of quantum mechanics and special relativity required the introduction of quantum field theory. The unification of magnetism and electricity led to electrodynamics, which was unified with the weak interactions into the electroweak interactions. There are good reasons to believe that the electroweak interactions and the strong interactions originate from the same underlying gauge theory: the grand unified theory. If general relativity is to be unified with a gauge theory, one first needs to understand how to unify general relativity and quantum mechanics, just as it was first necessary to understand how to unify quantum mechanics and special relativity before three of the forces of nature could be unified. The aim of this paper is much more modest - we want to understand some of the features of a quantum mechanical description of general relativity using some simple tools from quantum mechanics and general relativity. In particular, we shall show that if quantum mechanics and general relativity are valid theories of nature up to the Planck scale, they imply the existence of a minimal length in nature.

We shall address two questions: Is there a minimal length in nature, and is there a fundamental limit on the precision of distance measurement? The first question will be addressed in the second section while the second will be considered in the third section.

The usual approach to address the question of a minimal length is to do a scattering thought experiment [1-5], i.e. one studies the high energy regime of the scattering

\footnotetext{
a e-mail: xcalmet@gmail.com

b "New Talent" Presentation Award at the 44th Course of the International School of Subnuclear Physics, Erice, Italy, 29 Aug-7 Sep 2006
}

and finds that one cannot measure a length shorter than the Planck length. Here we shall argue that this is not enough to exclude a discreteness of spacetime with a lattice spacing shorter than the Planck length. The key new idea concerns how to define distance measurement more precisely and, in particular, the fact that such measurement actually involves two measurements.

We then apply our framework to the old thought experiment of Salecker and Wigner [6] and show that contractive states cannot beat the uncertainty due to quantu $\mathrm{m}$ mechanics for the measurement of a length. We then conclude.

\section{Minimal length from quantum mechanics and general relativity}

In this section we review the results obtained in $[7,8]$. We show that quantum mechanics and classical general relativity considered simultaneously imply the existence of a minimal length, i.e. no operational procedure exists that can measure a distance less than this fundamental length. The key ingredients used to reach this conclusion are the uncertainty principle from quantum mechanics and gravitational collapse from classical general relativity.

A dynamical condition for gravitational collapse is given by the hoop conjecture [9]: if an amount of energy $E$ is confined at any instant to a ball of size $R$, where $R<E$, then that region will eventually evolve into a black hole ${ }^{1}$. Although the hoop conjecture is, as its name says, a conjecture, it rests on firm footing. The least favorable case, i.e. as asymmetric as possible, is the one of two particles colliding

\footnotetext{
1 We use natural units where $\hbar, c$ and Newton's constant (or $\left.l_{\mathrm{P}}\right)$ are unity. We also neglect numerical factors of order one.
} 
head on. It has been shown that even in that case, when the hoop conjecture is fulfilled, a black hole is formed $[10,11]$.

From the hoop conjecture and the uncertainty principle, we immediately deduce the existence of a minimum ball of size $l_{\mathrm{P}}$. Consider a particle of energy $E$ which is not already a black hole. Its size $r$ must satisfy

$$
r \gtrsim \max [1 / E, E]
$$

where $\lambda_{\mathrm{C}} \sim 1 / E$ is its Compton wavelength and $E$ arises from the hoop conjecture. Minimization with respect to $E$ results in $r$ of order unity in Planck units or $r \sim l_{\mathrm{P}}$. If the particle $i s$ a black hole, then its radius grows with mass: $r \sim E \sim 1 / \lambda_{\mathrm{C}}$. This relationship suggests that an experiment designed (in the absence of gravity) to measure a short distance $l \ll l_{\mathrm{P}}$ will (in the presence of gravity) only be sensitive to scales $1 / l$.

Let us give a concrete model of minimum length. Let the position operator $\hat{x}$ have discrete eigenvalues $\left\{x_{i}\right\}$, with the separation between eigenvalues either of order $l_{\mathrm{P}}$ or smaller. (For regularly distributed eigenvalues with a constant separation, this would be equivalent to a spatial lattice, as seen in Fig. 1.) We do not mean to imply that in Nature a minimum length is realized in this particular fashion - most likely, the physical mechanism is more complicated and may involve, for example, spacetime foam or strings. However, our concrete formulation lends itself to detailed analysis. We show below that this formulation cannot be excluded by any gedanken experiment, which is strong evidence for the existence of a minimum length.

Quantization of position does not by itself imply quantization of momentum. Conversely, a continuous spectrum of momentum does not imply a continuous spectrum of position. In a formulation of quantum mechanics on a regu-

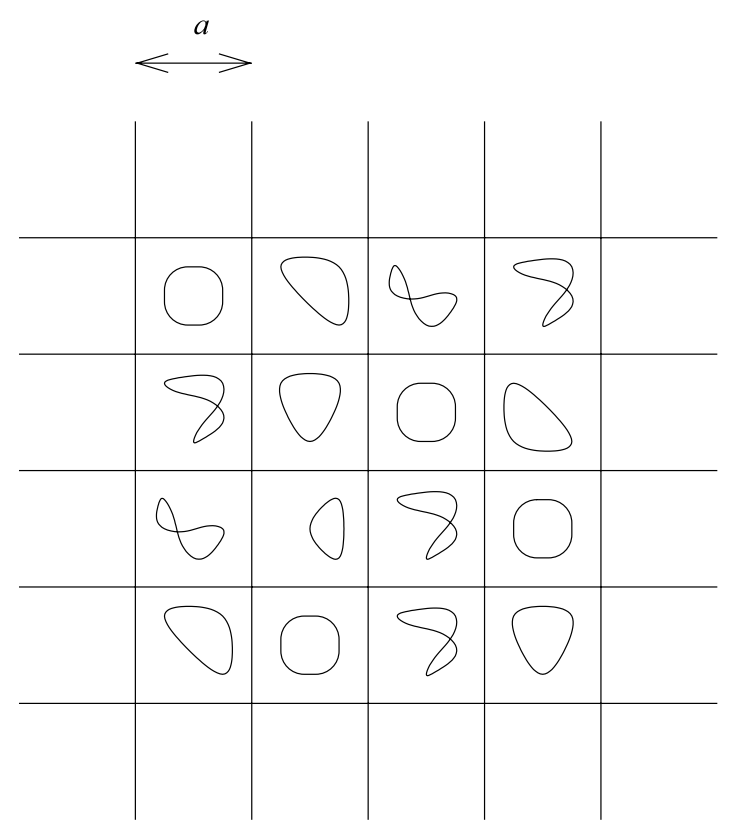

Fig. 1. We choose a spacetime lattice of spacing $a$ of the order of the Planck length or smaller. This formulation does not depend on the details of quantum gravity lar spatial lattice, with spacing $a$ and size $L$, the momentum operator has eigenvalues which are spaced by $1 / L$. In the infinite volume limit the momentum operator can have continuous eigenvalues even if the spatial lattice spacing is kept fixed. This means that the displacement operator

$$
\hat{x}(t)-\hat{x}(0)=\hat{p}(0) \frac{t}{M},
$$

(where $t$ is the time of the measurement and $M$ the mass of the system under consideration) does not necessarily have discrete eigenvalues (the right hand side of (2) assumes free evolution; we use the Heisenberg picture throughout). Since the time evolution operator is unitary, the eigenvalues of $\hat{x}(t)$ are the same as $\hat{x}(0)$. Importantly, though, the spectrum of $\hat{x}(0)$ (or $\hat{x}(t))$ is completely unrelated to the spectrum of the $\hat{p}(0)$, even though they are related by $(2)$. A measurement of arbitrarily small displacement (2) does not exclude our model of minimum length. To exclude it, one would have to measure a position eigenvalue $x$ and a nearby eigenvalue $x^{\prime}$, with $\left|x-x^{\prime}\right| \ll l_{\mathrm{P}}$.

Many minimum length arguments are obviated by the simple observation of the minimum ball. However, the existence of a minimum ball does not by itself preclude the localization of a macroscopic object to very high precision. Hence, one might attempt to measure the spectrum of $\hat{x}(0)$ through a time of flight experiment in which wavepackets of primitive probes are bounced off of well-localised macroscopic objects. Disregarding gravitational effects, the discrete spectrum of $\hat{x}(0)$ is in principle obtainable this way. But detecting the discreteness of $\hat{x}(0)$ requires wavelengths comparable to the eigenvalue spacing. For eigenvalue spacing comparable or smaller than $l_{\mathrm{P}}$, gravitational effects cannot be ignored because the process produces minimal balls (black holes) of size $l_{\mathrm{P}}$ or larger. This suggests that a direct measurement of the position spectrum to accuracy better than $l_{\mathrm{P}}$ is not possible. The failure here is due to the use of probes with very short wavelength.

A different class of instrument, the interferometer, is capable of measuring distances much smaller than the size of any of its sub-components. Nevertheless, the uncertainty principle and gravitational collapse prevent an arbitrarily accurate measurement of eigenvalue spacing. First, the limit from quantum mechanics - consider the Heisenberg operators for position $\hat{x}(t)$ and momentum $\hat{p}(t)$ and recall the standard inequality

$$
(\Delta A)^{2}(\Delta B)^{2} \geq-\frac{1}{4}(\langle[\hat{A}, \hat{B}]\rangle)^{2} .
$$

Suppose that the position of a free test mass is measured at time $t=0$ and again at a later time. The position operator at a later time $t$ is

$$
\hat{x}(t)=\hat{x}(0)+\hat{p}(0) \frac{t}{M} .
$$

We assume a free particle Hamiltonian here for simplicity, but the argument can be generalized $[7,8]$. The commutator between the position operators at $t=0$ and $t$ is

$$
[\hat{x}(0), \hat{x}(t)]=\mathrm{i} \frac{t}{M}
$$


so using (3) we have

$$
|\Delta x(0)||\Delta x(t)| \geq \frac{t}{2 M} .
$$

We see that at least one of the uncertainties $\Delta x(0)$ or $\Delta x(t)$ must be larger than of order $\sqrt{t / M}$. As a measurement of the discreteness of $\hat{x}(0)$ requires two position measurements, it is limited by the greater of $\Delta x(0)$ or $\Delta x(t)$, that is, by $\sqrt{t / M}$,

$$
\Delta x \equiv \max [\Delta x(0), \Delta x(t)] \geq \sqrt{\frac{t}{2 M}},
$$

where $t$ is the time over which the measurement occurs and $M$ the mass of the object whose position is measured. In order to push $\Delta x$ below $l_{\mathrm{P}}$, we take $M$ to be large. In order to avoid gravitational collapse, the size $R$ of our measuring device must also grow such that $R>M$. By causality, however, $R$ cannot exceed $t$. Any component of the device a distance greater than $t$ away cannot affect the measurement, hence we should not consider it part of the device. These considerations can be summarized in the inequalities

$$
t>R>M \text {. }
$$

Combined with (7), they require $\Delta x>1$ in Planck units, or

$$
\Delta x>l_{\mathrm{P}} .
$$

Notice that the considerations leading to (7), (8) and (9) were in no way specific to an interferometer, and hence are device independent. We repeat: no device subject to quantum mechanics, gravity and causality can exclude the quantization of position on distances less than the Planck length.

It is important to emphasize that we are deducing a minimum length which is parametrically of order $l_{\mathrm{P}}$, but may be larger or smaller by a numerical factor. This point is relevant to the question of whether an experimenter might be able to transmit the result of the measurement before the formation of a closed trapped surface, which prevents the escape of any signal. If we decrease the minimum length by a numerical factor, the inequality (7) requires $M \gg R$, so we force the experimenter to work from deep inside an apparatus which has far exceeded the criteria for gravitational collapse (i.e. it is much denser than a black hole of the same size $R$ as the apparatus). For such an apparatus a horizon will already exist before the measurement begins. The radius of the horizon, which is of order $M$, is very large compared to $R$, so that no signal can escape.

An implication of our result is that there may only be a finite number of degrees of freedom per unit volume in our universe - no true continuum of space or time. Equivalently, there is only a finite amount of information or entropy in any finite region of our universe.

One of the main problems encountered in the quantization of gravity is a proliferation of divergences coming from short distance fluctuations of the metric (or graviton). However, these divergences might only be artifacts of perturbation theory: minimum length, which is itself a non-perturbative effect, might provide a cutoff which removes the infinities. This conjecture could be verified by lattice simulations of quantum gravity (for example, in the Euclidean path integral formulation), by checking to see if they yield finite results even in the continuum limit.

\section{Limits on the measurement of large distances from fundamental physics}

In the section, we study whether quantum mechanics and general relativity can limit the precision of a distance measurement. In order to address this question we shall reconsider the thought experiment first proposed by Salecker and Wigner almost 50 years ago. In order to measure the distance $l$ we shall consider a clock which emits a light ray at a time $t=0$. The clock will suffer a recoil from the emission of the light ray which induces a position uncertainty in the position of the clock $x(0)$. The mirror which is at a distance $l$ from the clock will reflect the light ray, which is reabsorbed at a time $t$ at $x(t)$ by the clock, and again there is a recoil effect and the position of the clock will have some uncertainty (see Fig. 2).

Consider the Heisenberg operators for position $\hat{x}(t)$ and momentum $\hat{p}(t)$ and recall the standard inequality

$$
(\Delta A)^{2}(\Delta B)^{2} \geq-\frac{1}{4}(\langle[\hat{A}, \hat{B}]\rangle)^{2} .
$$

Suppose that the position of a free test mass is measured at time $t=0$ and again at a later time. The position operator at a later time $t$ is

$$
\hat{x}(t)=\hat{x}(0)+\hat{p}(0) \frac{t}{M} .
$$

The commutator between the position operators at $t=0$ and $t$ is

$$
[\hat{x}(0), \hat{x}(t)]=\mathrm{i} \frac{t}{M}
$$

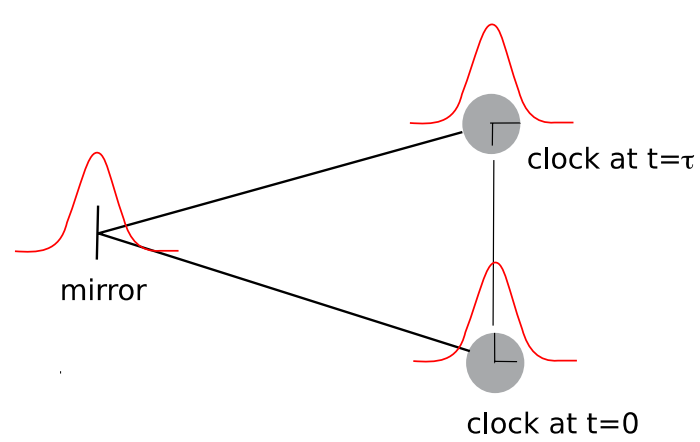

Fig. 2. Salecker and Wigner thought experiment to measure a length. A clock emits a light beam at a time $t=0$ which is reflected by a mirror and reabsorbed by the clock at a later time $t=\tau$. Quantum mechanics implies a spread of the wave function of the clock and of the mirror 
so using (10) we have

$$
|\Delta x(0)||\Delta x(t)| \geq \frac{t}{2 M} .
$$

Since the total uncertainty for the measurement of the distance $l$ is given by the sum of the uncertainties of $x(0)$ and $x(t)$, we find:

$$
\delta l \sim \sqrt{\frac{t}{2 M}} .
$$

Note that we are not forced to take the mass of the clock to be large, as in the previous section. There are actually two options, one of which is to allow the mass of the clock to grow at the same rate as $t$, the time necessary for the measurement, in which case we have

$$
\delta l \sim 1,
$$

or

$$
\delta l \sim l_{\mathrm{p}}
$$

The other option is to consider a fixed, finite, mass. This case applies to, e.g., the measurement of a distance performed with an interferometer such as LIGO [12]. The mass is at most the mass of the region of spacetime which feels one wavelength of the gravitational wave. In that case, the standard quantum limit [13-15] applies, and this is the well-known statement that LIGO operates at the standard quantum limit. Note that contractive states [16-18] cannot help to beat the standard quantum limit in a parametric manner. Again, as in $[7,8]$, the reason is that we need two measurements. Contractive states allow to make $\delta x(t)$ very small at the price of losing all the information about the uncertainty of $x(0)$ (see Appendix).

\section{Conclusions}

In this work we have shown that quantum mechanics and classical general relativity considered simultaneously imply the existence of a minimal length, i.e. no operational procedure exists that can measure a distance less than this fundamental length. The key ingredients used to reach this conclusion are the uncertainty principle from quantum mechanics and gravitational collapse from classical general relativity. Furthermore, we have shown that contractive states cannot be used to beat the limit obtained by Salecker and Wigner on the precision of a length measurement. Note that in that case we are not forced to consider very massive objects, and thus the gravitational collapse condition does not necessarily provide a bound. If we are forced to consider very massive objects, then the best precision that can be achieved for the measurement of a length is the minimal length itself. Our results have deep consequences for the detectability of quantum foam using astrophysical sources [19-26]. This, however, goes beyond the scope of this paper and shall be considered elsewhere.
Acknowledgements. I would like to thank G. 't Hooft and A. Zichichi for the wonderful Erice Summer School on Subnuclear Physics they have organized and where part of this work was done. It is with great pleasure that I acknowledge that the results of the third section were worked out during this summer school with S. Hsu. I am very grateful to A. Zichichi for the financial support that made my participation to this school possible as well as to H. Fritzsch who had nominated me for this school. Finally, I would like to thank F.R. Klinkhamer for enlightening discussions and for drawing my attention to the work of Salecker and Wigner. I would like to thank M. Ozawa for a helpful communication and for sending me a copy of his work on contractive states. This work was supported in part by the IISN and the Belgian science policy office (IAP V/27).

\section{Appendix : Contractive states}

Here we briefly review contractive states, following Ozawa's original work [18]. One introduces the operator $\hat{a}$ defined by

$$
\hat{a}=\sqrt{\frac{m \omega}{2 \hbar}} \hat{x}+\sqrt{\frac{1}{2 \hbar m \omega}} \mathrm{i} \hat{p} .
$$

The quantization of $\hat{x}$ and $\hat{p}$ implies $\left[\hat{a}, \hat{a}^{\dagger}\right]=1$. The parameter $\omega$ is free. The twisted coherent state $|\mu \nu \alpha \omega\rangle$ is the eigenstate of $\mu \hat{a}+\nu \hat{a}^{\dagger}$ with eigenvalue $\mu \hat{\alpha}+\nu \hat{\alpha}^{\star}$. The normalization of the wave function implies $|\mu|^{2}-|\nu|^{2}=1$. The free Hamiltonian is given by

$$
\hat{H}=\hat{p} / 2 m=\hbar \omega / 2\left(\hat{a}^{\dagger} \hat{a}+\frac{1}{2}-\frac{1}{2} \hat{a}^{2}-\frac{1}{2} \hat{a}^{\dagger 2}\right)
$$

and the wave function of this state is given by

$$
\begin{aligned}
\langle x \mid \mu \nu \alpha \omega\rangle= & \left(\frac{m \omega}{\pi \hbar|\mu-\nu|^{2}}\right)^{1 / 4} \\
& \times \exp \left(-\frac{m \omega}{\pi \hbar} \frac{1+2 \xi \mathrm{i}}{|\mu-\nu|^{2}}\left(x-x_{0}\right)^{2}+\mathrm{i} p_{0}\left(x-x_{0}\right)\right),
\end{aligned}
$$

with $\xi=\operatorname{Im}\left(\mu^{\star} \nu\right), \quad \alpha=(m \omega / 2 \hbar)^{1 / 2} x_{0}+1 /(2 \hbar m \omega)^{1 / 2} \mathrm{i} p_{0}$ where $x_{0}$ and $p_{0}$ are real. The position fluctuation for a free-mass is given by:

$$
\Delta x(t)^{2}=\frac{1}{4 \xi} \frac{\hbar \tau}{m}+\frac{2 \hbar}{m \omega}\left(|\mu+\nu| \frac{\omega}{2}\right)^{2}(t-\tau)^{2},
$$

with

$$
\tau=\xi \hbar m / \Delta p(0)^{2} .
$$

When $\xi>0$ the $x$-dependent phase leads to a narrowing of $\Delta x(t)$ compared to $\Delta x(0)$. States with the property $\xi>0$ are called contractive states. The absolute minimum is achieved for a time $\tau$ given by

$$
\tau=\frac{2 \xi}{\left.\omega|\mu+\nu|^{2}\right)}=\frac{\xi \hbar m}{\Delta p(0)^{2}}
$$


and one obtains

$$
\Delta x(\tau)=\frac{\hbar}{2 \Delta p(0)}=\frac{\Delta x(0)}{\sqrt{1+4 \xi^{2}}} .
$$

The price to pay to make $\Delta x(\tau)$ very small, i.e. smaller than the Planck length, for example, is to pick $\xi$ very large, which implies that $\tau$ is very large and thus $\Delta x(0)^{2}$ is very large as well. Keeping in mind that the measurement of a distance implies two measurements, we see that it is not possible to parametrically make the uncertainty in the measurement of a distance arbitrarily small. This is equivalent to the statement that LIGO and similar interferometers operate at the quantum limit - one can beat by some small factor the standard quantum limit, but it cannot be beaten parametrically.

\section{References}

1. L.J. Garay, Int. J. Mod. Phys. A 10, 145 (1995) [arXiv:grqc/9403008]

2. C.A. Mead, Phys. Rev. B 135, 849 (1964)

3. T. Padmanabhan, Class. Quantum Grav. 4, L107 (1987)

4. K. Fredenhagen et al., Commun. Math. Phys. 172, 187 (1995)

5. K. Fredenhagen et al., Phys. Lett. B 331, 33 (1994)

6. H. Salecker, E.P. Wigner, Phys. Rev. 109, 571 (1958)

7. X. Calmet, M. Graesser, S.D.H. Hsu, Phys. Rev. Lett. 93, 211101 (2004) [arXiv:hep-th/0405033]

8. X. Calmet, M. Graesser, S.D.H. Hsu, Int. J. Mod. Phys. D 14, 2195 (2005) [arXiv:hep-th/0505144]
9. K.S. Thorne, Nonspherical gravitational collapse: A short review. In: Magic Without Magic, ed. by J.R. Klauder (W.H. Freemann, San Francisco, 1972) pp. 231-258

10. D.M. Eardley, S.B. Giddings, Phys. Rev. D 66, 044011 (2002) [arXiv:gr-qc/0201034]

11. S.D.H. Hsu, Phys. Lett. B 555, 92 (2003) [arXiv:hepph/0203154].

12. http://www.ligo.caltech.edu/

13. V.B. Braginsky, Y.I. Vorontsov, Usp. Fiz. Nauk. 114, 41 (1974)

14. V.B. Braginsky, Y.I. Vorontsov, Sov. Phys. Uspekhi 17, 644 (1975)

15. C.M. Caves, Phys. Rev. Lett. 54, 2465 (1985)

16. H.P. Yuen, Phys. Rev. Lett. 51, 719 (1983)

17. H.P. Yuen, Phys. Rev. Lett. 52, 788 (1984)

18. M. Ozawa, Realization of Measurement and the Standard Quantum Limit, Squeezed and Nonclassical Light. In: Squeezed and Nonclassical Light, ed. by P. Tombesi, E.R. Pike (Plenum, New York, 1989), pp. 263-286

19. Y.J. Ng, H. van Dam, Phys. Lett. B 477, 429 (2000) [arXiv:gr-qc/9911054]

20. Y.J. Ng, H. van Dam, Found. Phys. 30, 795 (2000) [arXiv: gr-qc/9906003]

21. G. Amelino-Camelia, T. Piran, Phys. Lett. B 497, 265 (2001) [arXiv:hep-ph/0006210]

22. R. Lieu, L.W. Hillman, arXiv:astro-ph/0211402

23. R. Aloisio, P. Blasi, A. Galante, P.L. Ghia, A.F. Grillo, Astropart. Phys. 19, 127 (2003) [arXiv:astro-ph/0205271]

24. Y.J. Ng, H. van Dam, W.A. Christiansen, Astrophys. J. 591, L87 (2003) [arXiv:astro-ph/0302372]

25. R. Lieu, L.W. Hillman, Astrophys. J. 585, L77 (2003) [arXiv:astro-ph/0301184]

26. W.A. Christiansen, Y.J. Ng, H. van Dam, Phys. Rev. Lett. 96, 051301 (2006) [arXiv:gr-qc/0508121] 